\title{
The Effect of Obesity and Overweightness on Age of Menopausal
}

\section{Females}

\author{
Shahla Chaichian, ${ }^{1,2}$ Abolfazl Mehdizadehkashi, ${ }^{3,}$ Abdolreza Pazouki, ${ }^{4}$ Zhaleh Fazel, ${ }^{1}$ Yousef \\ Alimohamadi, ${ }^{2}$ Meisam Akhlaghdoust, ${ }^{2}$ and Fariborz Khorsandravan ${ }^{2}$ \\ ${ }^{1}$ Minimally Invasive Techniques Research Center in Women, Tehran Medical Sciences Branch, Islamic Azad University, Tehran, Iran \\ ${ }^{2}$ Pars Advanced and Minimally Invasive Medical Manners Research Center, Pars Hospital, Tehran, Iran \\ ${ }^{3}$ Endometriosis and Gynecologic Disorders Research Center, Iran University of Medical Sciences, Tehran, Iran \\ ${ }^{4}$ Minimally Invasive Surgery Research Center, Iran University of Medical Sciences, Tehran, Iran \\ "Corresponding author: Dr. Abolfazl Mehdizadehkashi, Endometriosis and Gynecologic Disorders Research Center, Iran University of Medical Sciences, Tehran, Iran. E-mail: \\ amehdizadehkashi@yahoo.com
}

Received 2017 April 26; Revised 2017 June 24; Accepted 2017 August 19.

\begin{abstract}
Background: The annual increase in the prevalence of obesity around the world highlights the importance of comorbidities. Along with hormonal changes induced by increased body mass index (BMI), age at menopause might also affect female's health profoundly. Thus, the present study aimed at studying the association between obesity and overweightness on age of menopause in females.

Methods: In this study, patients, who were referred to the obesity clinic of Rasoule-Akram hospital, during years 2011 and 2013 were recruited and the required information were extracted from the center's database. Inclusion criteria consisted of high BMI, no history of hysterectomy, genital tract cancer, no history of radiation to the abdomen or pelvis, and no primary amenorrhea. For analysis, analysis of variance (ANOVA) and Scheffe's post hoc test for multiple comparisons considering $\alpha$ of 0.05 for significance level were used and all data were analyzed by the SPSS version 22 software.

Results: The mean age of menopause was $51.6 \pm 6$ years and the majority of obese females had normal menopause age (57.6\%). There was no significant association between the patients BMI and age of menopause and it seems that obesity and overweightness had no effect on age of menopause $(\mathrm{P}=0.2)$.

Conclusions: It seems that overweightness or obesity was not associated with age of menopause, so it could not be said that females, who were overweight or obese compared to normal-weight females had earlier or later menopause.
\end{abstract}

Keywords: Menopause, Onset Age, Obesity

\section{Background}

The increased prevalence of obesity and overweightness in the recent years has highlighted the importance of obesity and its associated chronic diseases, such as type 2 diabetes mellitus (T2DM), hypertension, and cardiac diseases (1). In addition, obesity has negative impacts on multiple organs and systems of the body, such as the reproductive system, and is associated with increased risk of disorders in this system, such as polycystic ovarian syndrome (PCOS) (2), which is considered a frequent cause of anovulation in females with increased Body Mass Index(BMI) and Waist Circumference (WC) that may even remain after BMI correction (3).

Abdominal fat accumulation is a marker of metabolic disorder, similar to insulin resistance, and hyperinsulinemia leads to anovulation in females with obesity by increased androgen secretion of ovaries (4). Hormonal changes by overweightness and obesity could cause amen- orrhea and early menopause in females (5).

Menopause, defined as amenorrhea for more than a year without any physiological or pathological reason, is a natural phenomenon in female's life, during which menstrual cycle stops by a gradual decline in estrogen levels (6). An important issue in menopause is the age at menopause, and early menopause increases the risk of diseases, such as osteoporosis and cardiovascular diseases, and hence increases mortality rate in females (7). The normal age of menopause is considered 45 to 55 years old, yet this cutoff age is affected by several factors, such as smoking, consumption of oral contraceptives, educational level, and socio-economic status of the female (8). However, factors affecting the age of menopause in females have to be further elucidated. As race/ethnicity is one of the factors affecting age of menopause $(9,10)$, it is essential to study the normal age range of menopause and influential factors in Iran, where a higher number of females are reaching menopause, due to increased life expectancy and aging 
of the society. Among factors affecting age of menopause, BMI has been scarcely studied in Iran and given the importance of health consequences of age of menopause, the present study aimed at studying the normal age of menopause in an Iranian population and its association with obesity and overweightness.

\section{Methods}

\subsection{Study Design}

In this cross-sectional study, patients, who referred to the obesity clinic of minimally invasive surgery research center, Rasoul-e-Akram hospital, Tehran, Iran, during years 2011 to 2013, were selected as the target population of the study. Inclusion criteria consisted of high BMI, no history of hysterectomy, genital tract cancer, no history of radiation to the abdomen or pelvis, and no primary amenorrhea. Body mass index (BMI) of $\geq 35 \mathrm{~kg} / \mathrm{m}^{2}$ was considered severely obese and $\geq 40 \mathrm{~kg} / \mathrm{m}^{2}$ was considered very severely obese. Premature ovarian failure (POF) was considered as cessation of menstruation before the age of 40 , early menopause was defined as cessation of menstruation before the age of 45 , and late menopause was defined as menopause over the age of 55 years in females.

Eligible patients were recruited in the study based on the convenient sampling method and signed the written informed consent, after being clarified about the objectives of the study.

To gather the required information, variables including age, BMI, smoking, history of infertility, gravidity, parity, and number of live births, as well as age of menopause were extracted from the center's database.

After collecting the data and entering in the SPSS software version 22, descriptive statistics and analytical methods were used for analysis, including ANOVA for comparison of groups. Also, the researchers used Scheffe's post hoc test for multiple comparison with considering $\alpha: 0.05$ for significance level.

\section{Results}

In this study, 231 females aged 40 to 72 years were studied. The mean and standard deviation (SD) of age of participants was $54.6 \pm 6.7$ years, with mean BMI of $41.5 \pm 8.4$ $\mathrm{kg} / \mathrm{m}^{2}$ at the time of the study. Most females had reached menopause at normal age of menopause and mean age of menopause was $51.6 \pm 6$ years. Results of the prevalence and incidence of menopause are summarized in Table 1.

The results indicated that 23 patients (9.9\%) were current smokers or former smokers and 29 females (12.5\%) had a history of infertility and sterility.
Table 1. The Ratio of Different Menopause Categories in the Study Population

\begin{tabular}{lcc}
\hline Variables & No. & Mean of Age, \% \\
\hline Premature ovarian failure & 6 & 2.6 \\
\hline Early menopause & 56 & 24.2 \\
\hline Normal menopause & 133 & 57.6 \\
\hline Late menopause & 25 & 10.8 \\
\hline No menopause & 11 & 4.8 \\
\hline Total & 231 & 100 \\
\hline
\end{tabular}

As seen in Table 2, various classes of menopause showed significant differences in terms of pregnancy $(\mathrm{P}=$ 0.001). The groups had statistically significant differences in age of menopause $(P=0.01)$. However, differences in menopausal ages were not significantly different among different categories of $\mathrm{BMI}$ and parity $(\mathrm{P}=0.2$ and $\mathrm{P}=0.1)$.

The results of Scheffe's post hoc test showed that age of menopause was significantly different between premature ovarian failure and early menopause, normal menopause and late menopause $(\mathrm{P}<0.001)$. Other important results are shown in Table 3.

The results showed that the mean age of menopause was significantly different in females with irregular menstruation, while most of the females did not have this problem $(P=0.001)$. The age of menopause was significantly lower in females with menstrual abnormality than females with normal menstruation $(\mathrm{P}=0.01)$. The complete results are given in Table 3.

\section{Discussion}

Today, the association of obesity with various disorders has been well-known and it is assumed that it may also alter natural events in the body, such as menopause in females; while studies have scarcely addressed the effect of BMI on menopause. The present study aimed at investigating the age of menopause in females with overweightness and obesity, and the relationship between age of menopause and obesity.

Among 231 studied females, the results showed that mean and SD of age at menopause was $51.6 \pm 6$ years, which is within the normal range. In a previous study on 2000 Iranian females, age of menopause was reported as $50.4 \pm$ 4.3 years, which is in line with that of the present study (11), while age of menopause was reported as $47.13 \pm 3.9$ years in a similar study from Tehran (12), as well as another study from Shiraz ( $48.3 \pm 5.3$ years) (13) and Gorgan ( $47.6 \pm$ 4.45 years) (14), which were lower than that of the present study. This difference could be due to the differences in the 
Table 2. Comparison of Different Variables In Different Menopause Categories

\begin{tabular}{|c|c|c|c|c|c|}
\hline Variables & Premature Ovarian Failure $(\mathbf{n}=\mathbf{6})$ & Early Menopause $(\mathrm{N}=56$ ) & Normal Menopause $(\mathrm{N}=133)$ & Late Menopause ( $N=25)$ & PValue \\
\hline Age of menopause & $34 \pm 0.4$ & $44 \pm 0.5$ & $53 \pm 0.9$ & $60 \pm 2.1$ & 0.001 \\
\hline BMI & $47.4 \pm 7.3$ & $42.6 \pm 8.5$ & $41.1 \pm 8.2$ & $40.6 \pm 9.7$ & 0.200 \\
\hline Number of pregnancies & $2.6 \pm 1.2$ & $2.6 \pm 1.2$ & $3.4 \pm 1.6$ & $3.2 \pm 1.98$ & 0.010 \\
\hline Number of deliveries & $2 \pm 0.89$ & $2.2 \pm 0.98$ & $2.5 \pm 1.3$ & $2.6 \pm 1.6$ & 0.100 \\
\hline
\end{tabular}

Table 3. The Result of Scheffe's Post Hoc Test in Comparison of Age of Menopause and Number of Pregnancy Between Different Groups

\begin{tabular}{l|c|c}
\hline Age of Menopause & Compared with & P Value \\
\hline \multirow{2}{*}{$\begin{array}{l}\text { Premature ovarian failure (34 } \\
\pm 0.4)\end{array}$} & Early menopause $(44 \pm 0.5)$ & $<0.001$ \\
\cline { 2 - 3 } & Normal menopause $(53 \pm 0.9)$ & \\
\cline { 2 - 3 } & Late menopause $(60 \pm 2.1)$ & \\
\hline \multirow{2}{*}{$\begin{array}{l}\text { Early menopause }(44 \pm 0.5) \\
\text { Normal menopause }(53 \pm 0.9)\end{array}$} & Lormal menopause (53 \pm 0.9$)$ & \\
\cline { 2 - 3 } & Late menopause (60 \pm 2.1$)$ & $<0.001$ \\
\hline \multirow{2}{*}{ Number of pregnancies } & Early menopause (2.6 \pm 1.2$)$ & \\
\cline { 2 - 3 } & Normal menopause (3.4 \pm 1.6$)$ & 0.02 \\
\cline { 2 - 3 } & Late menopause (3.2 \pm 1.98$)$ & \\
\hline
\end{tabular}

inclusion criteria of the study population, as well as different characteristics of the participants, including mean age, age of onset of menstruation, etc. that effect the age of menopause. Further epidemiologic studies are required to set the normal age of menopause in Iranian females, as studies have merely focused on a selected sample in one center or city.

The relationship between obesity and infertility has been well demonstrated in many studies, and it seems that females with obesity are more likely to experience infertility through multiple mechanisms $(15,16)$. In the present study, $12.4 \%$ of participants had a history of infertility, which seems to be much higher than other Iranian reports $(17,18)$; this difference could be justified by the high BMI in these patients. Also, females with irregular menstrual cycles had different mean age of menopause, compared to females without irregular menstruation. It has been well-demonstrated that obese patients are prone to irregular menstrual pattern through hypothalamic hypogonadism and anovulation (19). Thus, obesity may indirectly effect the age of menopause, through irregular menstruation, although the results of the present study indicated no significant correlation between mean BMI and age of menopause in females with overweightness and obesity. Similar to these results, Davis et al. found that weight gain was not attributed to menopause tran- sition (20). Also, other researchers have found no association between BMI or physical activity and age at natural menopause (9), which are consistent with the results of the present study. Studies that have found an association between BMI and natural age of menopause have also determined its association with low BMI (21). However, as very few studies have focused on the effect of obesity on age of menopause and several factors have been determined to effect the age of menopause (10), further research is needed in this regard.

Another finding of the present study was the prevalence of smoking in the study population (9.9\%), which seems to be higher than the national statistics and is anticipated to increase with age. This can be due to the cooccurrence of smoking and obesity that have been previously demonstrated (22).

Also, hot flushes were not associated with menopause in the present study, while vasomotor symptoms were shown to be associated with BMI and age of menopause (23). This could be due to the retrospective data collection from medical records (database) that might have caused bias, as they were recorded based on patients' statement and not based on examination.

The present study's main strength was assessing the association of BMI and age of menopause that has scarcely been evaluated in Iran. However, it also had some limitations, including the retrospective nature of the study and data collection from medical records, as well as lack of a non-obese control group to reduce the effect of confounders on the study results. Moreover, selection of patients from one center and limited sample size reduce the generalizability of the results.

\subsection{Conclusion}

The results of the present study could not prove the significant effect of obesity on age of menopause, which has to be further studied, as long as age of menopause is affected by various factors that could vary among studies.

\section{References}

1. Al-Safi ZA, Polotsky AJ. Obesity and menopause. Best Pract Res Clin Obstet Gynaecol. 2015;29(4):548-53. doi: 10.1016/j.bpobgyn.2014.12.002. [PubMed: 25579233]. 
2. Castillo-Martinez L, Lopez-Alvarenga JC, Villa AR, Gonzalez-Barranco J. Menstrual cycle length disorders in 18- to 40-y-old obese women. Nutrition. 2003;19(4):317-20. doi: 10.1016/S0899-9007(02)00998-X. [PubMed: 12679164].

3. Lambert-Messerlian G, Roberts MB, Urlacher SS, Ah-Ching J, Viali S, Urbanek M, et al. First assessment of menstrual cycle function and reproductive endocrine status in Samoan women. Hum Reprod. 2011;26(9):2518-24. doi: 10.1093/humrep/der095. [PubMed: 21677061].

4. Deitel M, Stone E, Kassam HA, Wilk EJ, Sutherland DJ. Gynecologicobstetric changes after loss of massive excess weight following bariatric surgery. J Am Coll Nutr. 1988;7(2):147-53. doi: 10.1080/07315724.1988.10720232. [PubMed: 3361039].

5. Freeman EW, Sammel MD, Lin H, Gracia CR. Obesity and reproductive hormone levels in the transition to menopause. Menopause. 2010;17(4):718-26. doi: 10.1097/gme.0b013e3181cec85d. [PubMed: 20216473].

6. Landgren BM, Collins A, Csemiczky G, Burger HG, Baksheev L, Robertson DM. Menopause transition: Annual changes in serum hormonal patterns over the menstrual cycle in women during a nine-year period prior to menopause. J Clin Endocrinol Metab. 2004;89(6):2763-9. doi: 10.1210/jc.2003-030824. [PubMed: 15181055].

7. Shuster LT, Rhodes DJ, Gostout BS, Grossardt BR, Rocca WA. Premature menopause or early menopause: long-term health consequences. Maturitas. 2010;65(2):161-6. doi: 10.1016/j.maturitas.2009.08.003. [PubMed: 19733988].

8. Murugan A, Vanaja V. Evaluation of some risk factors on the age at menopause in south indian women. Int J Res Rev Phar Appl Sci. 2015;5(1):1117-24.

9. Gold EB, Bromberger J, Crawford S, Samuels S, Greendale GA, Harlow $\mathrm{SD}$, et al. Factors associated with age at natural menopause in a multiethnic sample of midlife women. Am J Epidemiol. 2001;153(9):865-74. doi: 10.1093/aje/153.9.865. [PubMed: 11323317].

10. Harlow BL, Signorello LB. Factors associated with early menopause. Maturitas. 2000;35(1):3-9. doi: 10.1016/S0378-5122(00)00092-X. [PubMed: 10802394].

11. Mohammad K, Sadat Hashemi SM, Farahani FK. Age at natural menopause in Iran. Maturitas. 2004;49(4):321-6. doi: 10.1016/j.maturitas.2004.02.006. [PubMed:15531128].

12. Nahidi F, Karman N, Vallaei N, Fazli Z. Studying incidence of menopause and its effective factors in Tehran [In Persian]. Pejouhesh. 2010;33(4):258-65.

13. Ayatollahi SM, Ghaem H, Ayatollahi SA. Sociodemographic factors and age at natural menopause in Shiraz, Islamic Republic of Iran. East Mediterr Health J. 2005;11(1-2):146-54. [PubMed: 16532683].

14. Abdollahi AA, Qorbani M, Asayesh H, Rezapour A, Noroozi M, Mansourian $\mathrm{M}$, et al. The menopausal age and associated factors in Gorgan, Iran. Med J Islam Repub Iran. 2013;27(2):50-6. [PubMed: 23741165].

15. Brewer CJ, Balen AH. The adverse effects of obesity on conception and implantation. Reproduction. 2010;140(3):347-64. doi: 10.1530/REP-090568. [PubMed: 20395425].

16. Jungheim ES, Travieso JL, Hopeman MM. Weighing the impact of obesity on female reproductive function and fertility. Nutr Rev. 2013;71 Suppl 1:S3-8. doi: 10.1111/nure.12056. [PubMed: 24147921].

17. Vahidi S, Ardalan A, Mohammad K. Prevalence of primary infertility in the Islamic Republic of Iran in 2004-2005. Asia Pac J Public Health. 2009;21(3):287-93. doi: 10.1177/1010539509336009. [PubMed: 19443880].

18. Kazem M, Ali A. An overview of the epidemiology of primary infertility in iran. J Reprod Infertil. 2009;10(3):213-6. [PubMed: 23926471].

19. Tortoriello DV, McMinn J, Chua SC. Dietary-induced obesity and hypothalamic infertility in female DBA/2J mice. Endocrinology. 2004;145(3):1238-47. doi:10.1210/en.2003-1406. [PubMed:14670988].

20. Davis SR, Castelo-Branco C, Chedraui P, Lumsden MA, Nappi RE, Shah D, et al. Understanding weight gain at menopause. Climacteric. 2012;15(5):419-29. doi: 10.3109/13697137.2012.707385. [PubMed: 22978257].

21. Ahuja M. Age of menopause and determinants of menopause age: A PAN India survey by IMS. J Midlife Health. 2016;7(3):126-31. doi: 10.4103/0976-7800.191012. [PubMed: 27721640].

22. Healton CG, Vallone D, McCausland KL, Xiao H, Green MP. Smoking, obesity, and their co-occurrence in the United States: cross sectional analysis. BMJ. 2006;333(7557):25-6. doi: 10.1136/bmj.38840.608704.80. [PubMed: 16698804].

23. Da Fonseca AM, Bagnoli VR, Souza MA, Azevedo RS, Couto Ede BJ, Soares JJ, et al. Impact of age and body mass on the intensity of menopausal symptoms in 5968 Brazilian women. Gynecol Endocrinol. 2013;29(2):116-8. doi: 10.3109/09513590.2012.730570. [PubMed: 23127175]. 